\title{
The Mu2e Experiment at Fermilab
}

\author{
Luca Morescalchi*i \\ University of Siena and INFN Pisa \\ E-mail: luca.morescalchiepi.infn.it
}

The Mu2e Experiment at Fermilab will search for the coherent, neutrinoless conversion of muons into electrons in the field of an aluminium nucleus with an unprecedented sensitivity. Such a charged lepton flavor-violating reaction probes new physics at a scale inaccessible with direct searches at either present or planned high energy colliders. Moreover, the Mu2e experiment both complements and extends the current search for the $\mu \rightarrow e \gamma$ decay at MEG and searches for new physics at the LHC. We will present the physics motivation for Mu2e, the experimental setup and the current status of the experiment.

XXIV International Workshop on Deep-Inelastic Scattering and Related Subjects

11-15 April, 2016

DESY Hamburg, Germany

\footnotetext{
* Speaker.

${ }^{\dagger}$ On the behalf of the Mu2e Collaboration, see [1].
} 


\section{Introduction}

Within the Standard Model (SM) heavy leptons decay in a way that almost perfectly conserves lepton family number. Even after the discovery of neutrino oscillations, in the minimal extension of SM, the predicted branching ratios of the Charged Lepton Flavor Violation (CLFV) processes in the muon sector are smaller than $10^{-50}$, unreachable by the current particle accelerators. Starting from the first search performed by Hinks and Pontecorvo in 1948 [2], despite repeated efforts, no CLFV process has been observed yet; so any experimental observation would be an unambiguous signal of physics beyond the Standard Model. A complete review that covers details of the CLFV experimental history, the theoretical implications and the technical iussues can be found in [3] and in [4]. One of the most promising processes for probing CLFV is the coherent muon conversion in the field of a nucleus, $\mu A \rightarrow e A$. In this process the nucleus is left intact and, due to the recoil, the resulting electron has a monochromatic energy slightly below the muon rest mass. The Mu2e experiment, concurrently with the COMET experiment being built at J-PARC [5], is designed to improve the current limit on the ratio $R_{\mu e}$ by 4 orders of magnitude over SINDRUM II [6]. The ratio $R_{\mu e}$ is defined as:

$$
R_{\mu e}=\frac{\Gamma\left(\mu^{-}+N(A, Z) \rightarrow e^{-}+N(A, Z)\right)}{\Gamma\left(\mu^{-}+N(A, Z) \rightarrow \text { all muon captures }\right)}
$$

where, in the Mu2e case, $N(A, Z)$ is an aluminum nucleus. Many New Physics scenarios, like many SUSYs, Leptoquarks, Heavy Neutrinos, GUTs, Extra Dimensions or Little Higgs, predict significantly enhanced values for $R_{\mu e}$, at a level accessible by the expected Mu2e sensitivity.
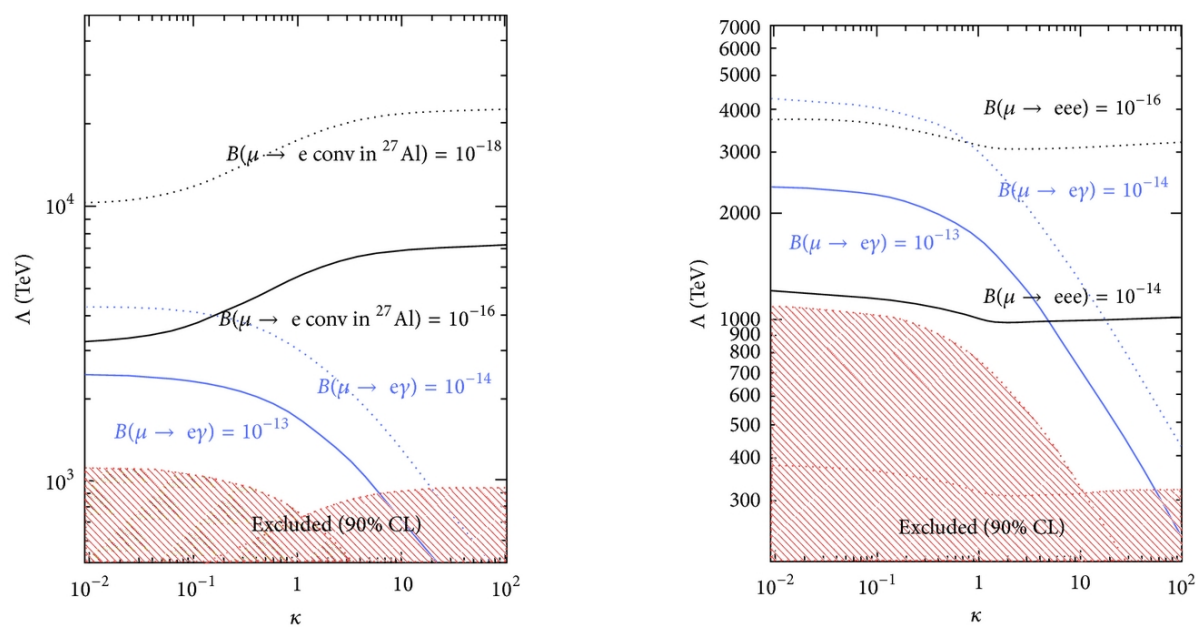

Figure 1: Left: Sensitivity of $\mu \rightarrow e \gamma$ (blue) and $\mu A \rightarrow e A$ conversion experiments (black) in the $(\kappa, \Lambda$ ) plane. Right: Sensitivity of $\mu \rightarrow e \gamma$ (blue) and $\mu \rightarrow e e e$ (black) in the same plane. The red regions are excluded by MEG for small $\kappa$ and by SINDRUM and SINDRUM II for large $\kappa$. [4]

To better understand the Mu2e discovery potential it is convenient to use a model-indipendent parametrization [4] that can describe CLFV processes as an admixture of two classes of diagrams: the first one includes magnetic moment loop diagrams with a photon exchanged; the second one 
includes both contact terms and the exchange of a new heavy particle. The coefficients of these two types of operators are parameterized by two independent constants: $\Lambda$, the mass scale of the new physics, and $\kappa$, a dimensionless parameter that mediates between the two terms. Figure 1 shows the exclusion plots at $90 \%$ of C.L. for several CLFV processes involving the muon in the $(\kappa, \Lambda)$ plane. Mu2e explores mass scales up to $10.000 \mathrm{TeV}$, far beyond the scales that will be accessible to direct observation at the LHC. Moreover, Mu2e and $\mu \rightarrow$ eee experiments are sensitive over a wide $\kappa$ range, while the $\mu \rightarrow e \gamma$ experiments, like MEG, are not sensitive in case the contact term dominates.

\section{The Mu2e Experiment}

To reach the sensitivity goal $R_{\mu e}<6 \cdot 10^{-17} @ 90 \%$ C.L., about $10^{18}$ stopped muons are needed. Muons are produced using $8 \mathrm{GeV}$ protons from the Fermilab accelerator complex, which provides a sequence of $200 \mathrm{nsec}$ wide micro-bunches separated by $1.7 \mu \mathrm{sec}$. The beam period is roughly twice the muon mean lifetime in Al nucleus, $\tau_{\mu}^{A l}=864 \mathrm{~ns}$ ). This particular beam structure, as shown in Figure 2, allows Mu2e to use a delayed selection windows to suppress the prompt background coming from proton interactions.

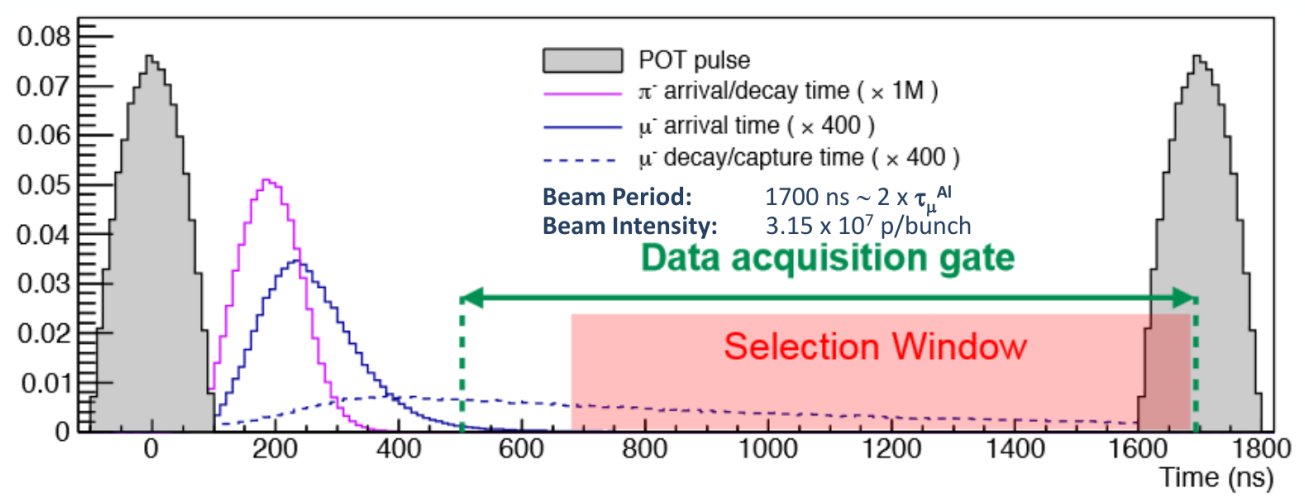

Figure 2: The Mu2e spill cycle for the proton on target pulse and the delayed selection window that allows an effective elimination of the prompt background.

The Mu2e apparatus consists of three superconductive solenoids, as shown in Figure 3: the Production Solenoid (PS), the Transport Solenoid (TS) and the Detector Solenoid (DS). The proton beam interacts in the PS with a tungsten target, producing mostly pions and muons. A back-scattered muon beam is captured by the PS and transported through the S-bend TS, that selects low momentum negative charged particles and delivers them to the aluminum stopping targets in the DS. Electrons from the $\mu \rightarrow e$ conversion (CE) in the stopping target are captured by the magnetic field in the DS and transported through the Straw Tube Tracker, that reconstructs the CE trajectory and its momentum. The $\mathrm{CE}$ then strikes the Electromagnetic Calorimeter, that provides independent measurements of the energy, the impact time and the position. Both detectors operate in a $10^{-4}$ Torr vacuum and in an uniform $1 \mathrm{~T}$ axial magnetic field. A cosmic ray veto system, which consists of four layers of scintillator bars, covers the entire DS and half of the TS, and guarantees a a veto inefficiency at the level of $10^{-4}$. Measurement of the total number of captured muons is provided 
by a high purity germanium detector, via the observation of the x-rays resulting from the nuclear capture. Additional details on the Mu2e apparatus can be found in [7].

\subsection{The Tracker}

Having the duty to identify the conversion electrons, the extremely low-mass tracker is the core of the experiment. It consists of about 20k straw tubes of $5 \mathrm{~mm}$ diameter filled with an $\mathrm{Ar} / \mathrm{CO}_{2}$ mixture. The tubes are arranged orthogonally to the solenoid axis and are grouped, over $3.2 \mathrm{~m}$, in 18 tracking stations that have the inner $38 \mathrm{~cm}$ left un-instrumented. Only particles with a $\mathrm{P}_{T}>55 \mathrm{MeV}$ reach the detector and only particles with $\mathrm{P}_{T}>90 \mathrm{MeV}$ leave enough hits to form a reconstructible trajectory. In this way, the tracking system is blind to the majority of the background particles coming from the muons interactions in the stopping target. The cluster of straw hits associated to a particle trajectory is identified using a robust helix fit algorithm to perform the pattern recognition. A Kalman based track fitter provides a precise geometric and kinematic estimate of the electron track parameters, resulting in a momentum resolution of about $200 \mathrm{keV} / \mathrm{c}$ for CE.

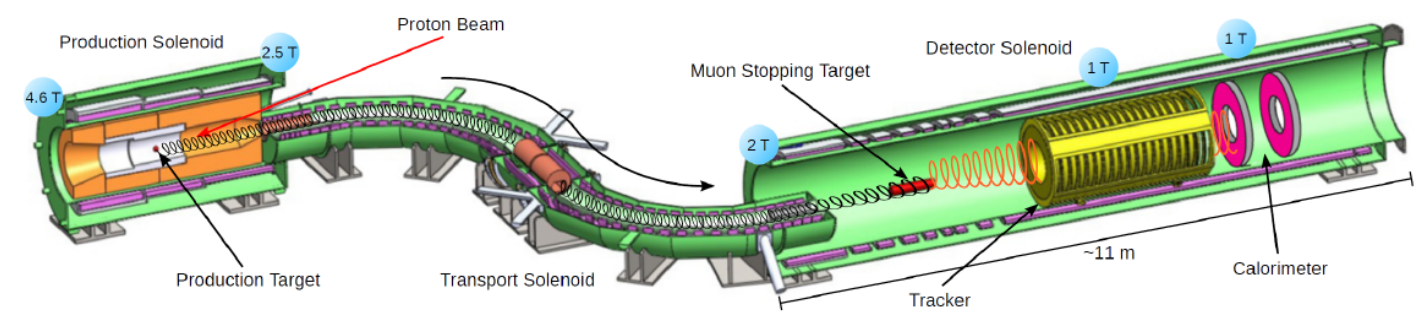

Figure 3: Diagram of the Mu2e muon beam-line and detector. The cosmic ray veto system and the stopping target monitor are not shown in this figure.

\subsection{The Electromagnetic Calorimeter}

The crystal calorimeter consists of about 1400 undoped $3.4 \times 3.4 \times 20 \mathrm{~cm}^{3}$ CsI crystals, arranged in two disks. The disks are separated by $75 \mathrm{~cm}$, half of the conversion electron $\lambda$, in order to maximize the calorimeter acceptance. Each crystal is coupled with two $2 \times 3$-arrays of $6 \times 6 \mathrm{~mm} \mathrm{UV}$-extended SiPM. This configuration ensures at $100 \mathrm{MeV}$ an energy resolution better than the $10 \%$ and a time resolution better than 500 ps. The calorimeter complements the tracker providing: a powerful particle identification, a seed for the pattern recognition in the tracker and an independent software trigger system. The particle identification provides a good separation between CEs and muons unvetoed by the CRV and mimicking the signal. The required muon rejection factor is provided with 95\% efficiency on the signal, combining the time of flight difference between the tracker track and the calorimeter cluster with the E/p ratio, see Figure 4, into a simple likelihood.

\section{Expected Background}

Muons that stop in the stopping target can undergo beta decay in the field of the nucleus (decay in orbit, DIO), be captured (ordinary muon capture, OMC) or convert to electrons. For Aluminium, the muon DIO probability is about $39 \%$. The kinematic limit for the muon decay in vacuum is at 

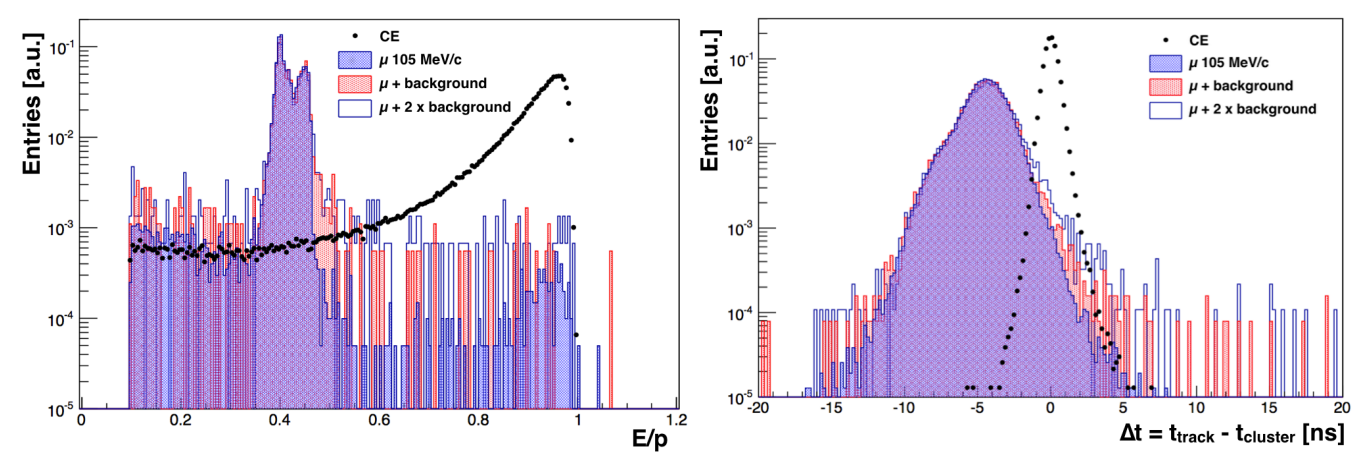

Figure 4: Distribution in $\Delta \mathrm{t}=\mathrm{t}_{\text {track }}-\mathrm{t}_{\text {cluster }}$, on the left, and $\mathrm{E} / \mathrm{p}$, on the right, for cosmic electrons and $105 \mathrm{MeV} / \mathrm{c}$ muons reconstructed as electrons. Different colors correspond to different assumption of the background level. [8]

about $54 \mathrm{MeV}$, but the recoil of the nucleus generates a long tail that has the endpoint exactly at the conversion electron energy. This tail falls rapidly down as $\left(E_{C E}-E\right)^{5}$ and only $10^{-17}$ of the DIO energy spectrum is within $1 \mathrm{MeV}$ on the left of the endpoint. This represents the main background source for Mu2e and scales linearly with the beam intensity. So the only technique to suppress DIO is to carefully measure the momentum with the high resolution tracker. The OMC, which has a probability of $61 \%$, is not a source of background but for a very small contribution from photons produced in radiative muon captures (RMC), as reported in Table 5. Backgrounds arising from interactions at the production target are overwhelmingly prompt and arrive at the stopping target in time with the muon beam. As shown in Figure 2, these backgrounds are eliminated by defining a delayed selection windows. However, out-of-time protons impinging on the production target can produce background that come anyway in the search window. A proton extinction factor of $10^{10}$ is needed to keep these Late Arrival Backgrounds (LAB) at a neglegible level. In Mu2e it is obtained including in the proton extraction line a custom AC dipole that sweeps out-of-time protons into a collimator. The remaining background sources consists of cosmic rays and antiprotons. Cosmic rays can interact in the DS producing delta rays or can be trapped directly in the DS magetic field and leave a track in the tracker equal to a conversion electron helix. The cosmic ray veto system ensures that the first type of cosmic rays background is kept under control, while the second component is suppressed by means of the particle identification capabilities of the detector system. Antiprotons from interaction in the PS can drift slowly until they annihilate. These annihilations produce high multiplicity final states that can generate background in time with the search window. To limit antiprotons induced background, two Be absorbers are placed at the entrance and in the middle of the TS. Table 5 summarizes the expected backgrounds yield for three years of data taking [7], corresponding to $6.8 \times 10^{17}$ stopped muons. The total estimated background yeld is 0.4 events and the expected sensitivity is $R_{\mu e}<6 \times 10^{-17}$ at $90 \%$ of C.L. .

\section{Status}

The Mu2e experiment has recently received the CD-3 approval from the U.S. Department of Energy. The R\&D program is complete and all the detectors are frozen as a final design. The first large scale prototypes are under construction and beam tests are scheduled for the fall 2016. The installation phase will begin in 2017, while data taking is expected to start in early 2021. 


\begin{tabular}{|llr}
\hline Category & Background process & $\begin{array}{r}\text { Estimated yield } \\
\text { (events) }\end{array}$ \\
\hline \hline Intrinsic & Muon decay-in-orbit (DIO) & $0.199 \pm 0.092$ \\
Late Arriving & Muon capture (RMC) & $0.000_{-0.000}^{+0.004}$ \\
& Pion capture (RPC) & $0.023 \pm 0.006$ \\
& Muon decay-in-flight ( $\mu$-DIF) & $<0.003$ \\
& Pion decay-in-flight ( $\pi$-DIF) & $0.001 \pm<0.001$ \\
Miscellaneous & Beam electrons & $0.003 \pm 0.001$ \\
& Antiproton induced & $0.047 \pm 0.024$ \\
& Cosmic ray induced & $0.092 \pm 0.020$ \\
\hline \hline
\end{tabular}

Figure 5: Backgrounds yield expected by Mu2e in three years of data taking, corresponding to $6.8 \times 10^{17}$ stopped muons. [7]

\section{Conclusions}

Mu2e will search for the CLFV process $\mu^{-} \mathrm{Al} \rightarrow \mathrm{e}^{-} \mathrm{Al}$ with an unprecedented sensitivity, improving by a factor 10000 the current best limit. If a signal is observed, that will be undeniable proof of new physics and it will provide data complementar to LHC and to the other CLFV experiments. If no signal is found, the expected upper sensitivity $R_{\mu e}<6 \times 10^{-17}$ will set constrains to many models at mass scale up to thousands of TeV.

\section{Acknowledgments}

We are grateful for the vital contributions of the Fermilab staff and the technical staff of the participating institutions. This work was supported by the US Department of Energy; the Italian Istituto Nazionale di Fisica Nucleare; the US National Science Foundation; the Ministry of Education and Science of the Russian Federation; the Thousand Talents Plan of China; the Helmholtz Association of Germany; and the EU Horizon 2020 Research and Innovation Program under the Marie Sklodowska-Curie Grant Agreement No.690385. Fermilab is operated by Fermi Research Alliance, LLC under Contract No. De-AC02-07CH11359 with the US Department of Energy.

\section{References}

[1] Mu2e Collaboration, http://mu2e.fnal.gov/collaboration.shtml

[2] E. P. Hincks and B. Pontecorvo, Phys. Rev. 73 (1948) 257. doi:10.1103/PhysRev.73.257

[3] R. H. Bernstein and P. S. Cooper, Phys. Rept. 532 (2013) 27 doi:10.1016/j.physrep.2013.07.002 [arXiv:1307.5787 [hep-ex]].

[4] F. Cei and D. Nicolo, Adv. High Energy Phys. 2014 (2014) 282915. doi:10.1155/2014/282915

[5] Y. G. Cui et al. [COMET Collaboration], KEK-2009-10.

[6] W. H. Bertl et al., Eur. Phys. J. C 47 (2006) 337. doi:10.1140/epjc/s2006-02582-x

[7] Mu2e Collaboration, Mu2e Technical Design Report, http://arxiv.org/abs/1501.05241, 2015

[8] G. Pezzullo, FERMILAB-THESIS-2016-02. 